\title{
Malaysian Version of the Sniffin' Sticks Identification Smell Test: Cutoff Points of Hyposmia
}

\author{
Salina Husain Irfan Affandi Hamid Farah Dayana Zahedi \\ Aneeza Khairiyah Wan Hamizan \\ Department of Otorhinolaryngology-Head and Neck Surgery, Faculty of Medicine, UKM Medical Centre, Cheras, \\ Kuala Lumpur, Malaysia
}

\section{Keywords}

Sniffin' Sticks · Identification test - Cutoff point · Hyposmia . Age

\begin{abstract}
Introduction: Cultural backgrounds can influence the smell test because of the unfamiliarity of different populations with a number of odorants. The study aims to determine the cutoff points for hyposmia using the cultural adaptation of the Malaysian version of the Sniffin' Sticks identification smell test among different age groups. Methods: In this cross-sectional study, healthy participants were recruited from a hospital, senior citizen homes, and schools from 2019 until 2021. Participants were divided into 5 different age groups (A: children, B: adolescents, C: young adults, D: middle-aged adults, and E: older adults) and underwent the identification smell test. Scores below the 10th percentile in these age groups were used to determine the cutoff points for hyposmia. Results: The cutoff points for the smell identification scores for hyposmia at the 10th percentile in children, adolescents, and adults (young, middle-aged, and older) were less than 6, 9, and 11, respectively. Pairwise comparisons were made using the Bonferroni post hoc test, with $p<0.001$. Scores showed a significant difference between
\end{abstract}

males (mean [SD]: 11.43 [2.42]) and females (12.01 [1.98]); $p=0.005$. Conclusion: Our study found that the cutoff points of the identification smell test for hyposmia were lower than those of other studies. It is important to use these cutoff points in our population to establish the correct integrity of olfaction function in the clinic setting and in future research.

(c) 2022 S. Karger AG, Basel

\section{Introduction}

Olfaction is one of the special senses used in daily life. Olfaction plays an important role in detecting food, and it is also crucial in avoiding harmful substances such as gas leaks, fire, and eating spoiled food. Therefore, olfactory impairment not only reduces quality of life but can also be life-threatening $[1,2]$.

Olfactory impairment can be classified as hyposmia (reduced sense of smell), anosmia (no sense of smell), parosmia (distortion of smell), and phantosmia (hallucination of smell) [3]. The prevalence of olfactory disorders ranges from $3.7 \%$ to $75 \%$, depending on age and the population evaluated [4]. Hyposmia accounts for $13-16 \%$ and anosmia for $5 \%[5,6]$.
Correspondence to:

Salina Husain,drsalina_h@ukm.edu.my 
Odor identification tests are commonly used in clinics. The Sniffin' Sticks smell identification test was developed by Hummel et al. [7] and has been validated in several European countries. The test is a common olfactory test used to evaluate the olfactory function of smell. The smell identification test relies on the individual's familiarity with both the odorant and the descriptors tested $[8,9]$. Normosmia is defined as an identification score $>10$ th percentile of the distribution of normal values of the study population according to age and sex. Hyposmia is defined as an identification score $<10$ th percentile of the distribution of normal values of the study population according to age and sex $[10,11]$. Evidence has shown that different age groups have different values of identification scores at the 10 th percentile. Previous researchers found that the values were lower in the youngest and oldest age groups [12].

The cultural adaptation of the Sniffin' Sticks identification test is recommended in countries with different cultural backgrounds because of unfamiliar odorants and their description or terms used $[8,13]$. In a previous study, the Malaysian version of the Sniffin' Sticks identification smell test was created [14]. However, a cutoff score for determining hyposmia/normosmia in the normal population using this tool needs to be determined. This study aimed to establish the cutoff points for hyposmia using a validated, culturally adapted Malaysian version of the Sniffin' Sticks identification smell test among different age groups in the Malaysian population.

\section{Methods}

This was a cross-sectional study involving healthy subjects in a hospital, senior citizen homes, and schools from 2019 until 2021. A total of 450 subjects were included who ranged in age from 7 to 80 years. They were divided into 5 different age groups: A, children, 7-12 years; B, adolescents, $13-17$ years; C, young adults, 1840 years; $\mathrm{D}$, middle-aged adults, $41-60$ years; and $\mathrm{E}$, older adults, 61-80 years.

The study was conducted in accordance with the Declaration of Helsinki for research on human participants. Written informed consent was obtained from all participants and legal guardians for participants younger than 18 years of age. We excluded participants with neurodegenerative disorders, congenital mucociliary clearance abnormality, neuropsychiatric disorder, recent upper respiratory tract infection within the past 2 weeks, sinonasal disease or malignancy, immunodeficiency diseases, autoimmune diseases, nasal surgery, or head trauma.

The olfactory assessment was conducted using the culturally adapted Malaysian version of the Sniffin' Sticks identification test. The original 16 items were used. However, 2 odorant descriptors and 12 distractors were changed to suit the local population's cultural and linguistic background. The tip of the pen was introduced about $2 \mathrm{~cm}$ in front of both nostrils for 2-3 s. Participants were then asked to identify the odorant from a list of 4 items (including 1 correct answer and 3 distractors). The odor identification process was repeated using 16 pens with different odors, with a time interval of 20-30 s between odor presentations.

\section{Statistical Analysis}

Data were analyzed using SPSS software (version 26). The descriptive statistic was used to establish the normative data for different age groups. The 10th percentile of the identification score was calculated to assess participants' olfactory performance. The data are mainly presented as mean (SD). We analyzed the smell identification scores between age groups using analysis of variance (ANOVA). Pairwise comparisons using Bonferroni-corrected multiple comparisons between the 5 age groups were advocated. Statistical significance was defined as $p<0.05$.

\section{Results}

The mean (SD) identification score for group A (children) was 9.26 (1.88), for group B (adolescents) was 11.13 (2.62), for group C (young adults) was 12.80 (1.41), for group D (middle-aged adults) was 13.06 (1.31), and for group E (older adults) was 12.60 (1.22). The children had a lower mean identification score compared with the other groups (Table 1).

Using descriptive analysis, we assessed the olfactory identification performance among the different age groups. The identification score in children, adolescents, and adults (young, middle-aged, and older) at the 10th percentile was 6,9 , and 11 , respectively. The lowest score was observed in children (Table 1). ANOVA showed a significant difference between age groups (Table 2). Pairwise comparisons using Bonferroni-corrected multiple comparisons showed a significant difference between children, adolescents, and adults (young, middle-aged, and older; $p<0.001$ ). There was no significant difference between young adults, middle-aged adults, and older adults $(p=1.000$; Table 3$)$. The identification score showed a significant difference between men and women ( $p=0.005)$, suggesting that women outperform men throughout the different age groups (Table 4).

\section{Discussion}

Olfactory dysfunction has attained great interest in recent years. Olfaction helps an individual detect and avoid health and environmental hazards such as fire, leaking natural gas, rotten food, and dead animals in the house.

A lower identification score indicates that the individual has a decreased ability for identifying odors. The cul- 
Table 1. Descriptive normative value obtained in healthy subjects using Malaysian version of the Sniffin' Sticks identification smell test

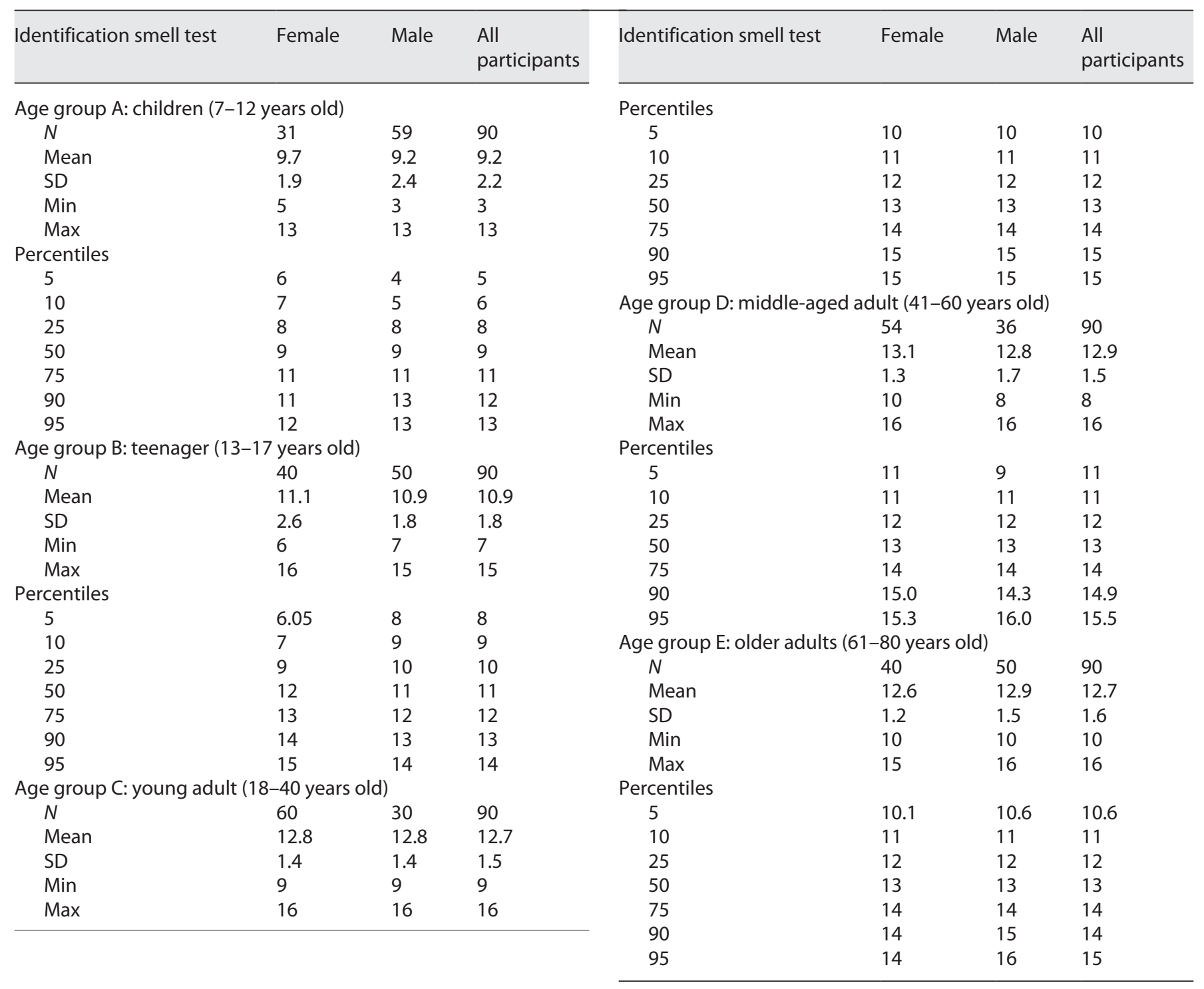

Table 2. Identification smell test scores and different age groups

\begin{tabular}{llllll}
\hline Identification smell test & Sum of squares & df & Mean square & $F$ & Sig \\
\hline Between groups & 938.956 & 4 & 234.739 & 81.397 & 0.000 \\
Within groups & $1,283.322$ & 445 & 2.884 & & \\
\hline Total & $2,222.278$ & 449 & & & \\
\hline
\end{tabular}

tural adaptation smell test is recommended in countries with multiple cultural backgrounds because of the population's unfamiliarity with the tested odors. To obtain a reliable identification score for normosmia or hyposmia among different age groups, normative data using the cultural adaptation smell test are critical to avoid misin- terpretation of test results, which could lead to an incorrect diagnosis.

A normative data study using a cultural adaptation of the Portuguese version of the Sniffin' Sticks smell test found that the cutoff points of identification scores for hyposmia in participants aged $16-55$ years and $>55$ years 
Table 3. Bonferroni-corrected multiple comparisons between the 5 age groups

\begin{tabular}{lllllll}
\hline \multirow{2}{*}{$\begin{array}{l}\text { Age } \\
\text { groups }\end{array}$} & $\begin{array}{l}\text { Age } \\
\text { groups }\end{array}$ & $\begin{array}{l}\text { Mean } \\
\text { difference }\end{array}$ & SE & Sig & \multicolumn{2}{l}{$95 \%$ confidence interval } \\
\cline { 6 - 7 } A & & & & \multicolumn{2}{l}{ lower bound } & upper bound \\
& B & $-1.700^{*}$ & 0.253 & 0.000 & -2.41 & -0.99 \\
& C & $-3.500^{*}$ & 0.253 & 0.000 & -4.21 & -2.79 \\
& D & $-3.733^{*}$ & 0.253 & 0.000 & -4.45 & -3.02 \\
& E & $-3.511^{*}$ & 0.253 & 0.000 & -4.23 & -2.80 \\
\hline B & A & $1.700^{*}$ & 0.253 & 0.000 & 0.99 & 2.41 \\
& C & $-1.800^{*}$ & 0.253 & 0.000 & -2.51 & -1.09 \\
& D & $-2.033^{*}$ & 0.253 & 0.000 & -2.75 & -1.32 \\
& E & $-1.811^{*}$ & 0.253 & 0.000 & -2.53 & -1.10 \\
\hline C & A & $3.500^{*}$ & 0.253 & 0.000 & 2.79 & 4.21 \\
& B & $1.800^{*}$ & 0.253 & 0.000 & 1.09 & 2.51 \\
& D & -0.233 & 0.253 & 1.000 & -0.95 & 0.48 \\
& E & -0.011 & 0.253 & 1.000 & -0.73 & 0.70 \\
\hline D & A & $3.733^{*}$ & 0.253 & 0.000 & 3.02 & 4.45 \\
& B & $2.033^{*}$ & 0.253 & 0.000 & 1.32 & 2.75 \\
& C & 0.233 & 0.253 & 1.000 & -0.48 & 0.95 \\
& E & 0.222 & 0.253 & 1.000 & -0.49 & 0.94 \\
\hline E & A & $3.511^{*}$ & 0.253 & 0.000 & 2.80 & 4.23 \\
& B & $1.811^{*}$ & 0.253 & 0.000 & 1.10 & 2.53 \\
& C & 0.011 & 0.253 & 1.000 & -0.70 & 0.73 \\
& D & -0.222 & 0.253 & 1.000 & -0.94 & 0.49 \\
\hline & & & & & &
\end{tabular}

* The mean difference is significant at the 0.05 level.
Table 4. The difference between mean identification score and gender

\begin{tabular}{lllll}
\hline Variable & Gender & Mean (SD) & $\begin{array}{l}\text { Mean difference } \\
(95 \% \mathrm{Cl})\end{array}$ & p value \\
\hline Identification smell scores & $\begin{array}{l}\text { Female } \\
\text { Male }\end{array}$ & $\begin{array}{llll}12.01(1.98) \\
11.43(2.42)\end{array}$ & $0.21(0.17,0.99)$ & 0.005 \\
\hline
\end{tabular}

were 13 and 10, respectively [15]. Vickers et al. [16] conducted normative data research of the Sniffin' Sticks odor identification test (SS-OIT) in adults. They found that age had a significant effect on SS-OIT. The average SS-OIT score of healthy adults aged 16-55+ years was between 12.06 and 13.68 [16]. A normative data study by Schriever et al. [17] using the Sniffin' Kids test found the various cutoff points for hyposmia at the 10th percentile in the different age groups. The cutoff identification scores in the age groups of 6-8 years, 9-14 years, and 15-17 years were 7,8 , and 10 , respectively [17]. Our study revealed that the lower cutoff identification scores compared with a Western population for participants aged 7-12 years, 13-17 years, and $18-80$ years were 6,9 , and 11 , respectively.
Sorokowska et al. [18] suggested that in children, both knowledge of odors and cognitive abilities influence olfactory performance. Olfactory identification is challenging in children because of the unfamiliarity of the odors, low concentration abilities, and insufficient verbal development. The ability to identify olfactory stimuli is associated with complex measures such as recognition, discrimination, retrieval of name by memory, language, and verbal skills [19]. It has been shown that olfactory performance is significantly influenced by individual's cognitive profile. Therefore, the level of development of cognitive abilities among different age groups will have an effect on the olfactory identification test. These factors may result in wrong responses to the tested odors, and the pediatric age group may be wrongly labeled with hyposmia [18]. 
Children require olfactory tests that suit their knowledge of odors and level of cognitive development. The tests must be of a short duration and easy to use in assessing their olfaction performance. Several olfactory tests in children have mainly focused on odor identification abilities, such as the Sniffin' Kids test, Smell Wheel, and Scratch and Sniff card system [17, 20, 21].

In a previous study, the Malaysian version of the Sniffin' Stick identification smell test was validated, and the test retest reliability was carried out [14]. For the current study, we divided the population into 5 groups to achieve the normative data for each age group with a cutoff value at the 10th percentile of the odor identification scores. We found that the identification scores in our population were lower than those in the Western population. The possible explanation for this fact is that genetics, ethnicity, and environment may also influence the identification of smell $[22,23]$.

We compared the findings of our study with the results of other studies that used the Sniffin' Sticks smell test. Most of the previous studies were conducted in very young children (3-6 years old) and adults $[18,24]$. The closest comparison we could find with the pediatric age group was a study by Schriever et al. [17], which used the Sniffin' Kids smell test. The test consists of 14 items, compared with the original Sniffin' Sticks test, which is composed of 16 items. Items such as apple and turpentine were removed because of significant lower scores in children [17]. Based on our previous cultural adaptation study, we have changed these descriptors to odors more familiar to our population.

A study involving a large sample size of 9,139 participants showed a significant difference in olfactory performance between sexes, in which women outperformed men $(p<0.001)$ [13]. Our study also found that women had significantly better olfactory performance compared with men $(p=0.005)$. Women also had a significantly higher ability of identifying odors.

We conclude that the cutoff points for hyposmia in children, adolescents, and adults using the cultural adaptation of the Malaysian version of the Sniffin' Sticks smell test were less than 6,9, and 11, respectively. We also found that females could identify odor better than males could.

\section{Acknowledgments}

We are thankful to the volunteers for their participation in the study. We also acknowledge the assistance of the doctors and staff members of UKM.

\section{Statement of Ethics}

Ethical approval for the study was obtained from the Research Ethics Committee of UKMMC (UKM PPI/111/8/JEP-2019-562). All the procedures were carried in accordance with the Declaration of Helsinki 1964. Written informed consent was obtained from participants and their parent/legal guardian to participate in the study.

\section{Conflict of Interest Statement}

The authors declare no conflicts of interest, and there is nothing to disclose about this study.

\section{Funding Sources}

The study was funded by UKM Fundamental Research Grant code of FF-2019-405.

\section{Author Contributions}

S.H.: conceptualization, data analysis, writing - original draft, and writing - review and editing. I.A.H.: conceptualization, data collection, and writing - review and editing. F.D.Z.: conceptualization, writing - original draft, and writing - review and editing. A.K.W.H.: conceptualization, data analysis, and writing - review and editing.

\section{Data Availability Statement}

All data generated or analyzed during this study are included in this article. Further enquiries can be directed to the corresponding author.

\section{References}

1 Doty RL, Kamath V. The influences of age on olfaction: a review. Front Psychol. 2014 Feb; $5: 20$.

2 Miwa T, Furukawa M, Tsukatani T, Costanzo RM, DiNardo LJ, Reiter ER. Impact of olfactory impairment on quality of life and disability. Arch Otolaryngol Head Neck Surg. 2001; 127:497-503.

3 Mainland JD, Barlow LA, Munger SD, Millar SE, Vergara MN, Jiang $P$, et al. Identifying treatments for taste and smell disorders: gaps and opportunities. Chem Senses. 2020;45: 493-502.

4 Fornazieri MA, dos Santos CA, Bezerra TFP, Pinna Fde R, Voegels RL, Doty RL. Development of normative data for the Brazilian adaptation of the University of Pennsylvania smell identification test. Chem Senses. 2015; 40:141-9.

5 Pinkaew B, Assanasen P, Bunnag C. Smell discrimination and identification scores in Thai adults with normosmia. Asian Biomed. 2015; 9:789-95. 
6 Brämerson A, Johansson L, Ek L, Nordin S, Bende M. Prevalence of olfactory dysfunction: the Skövde population-based study. Am Laryngol Rhinol Otol Soc. 2004;114: 733-7.

7 Hummel T, Sekinger B, Wolf SR, Pauli E, Kobal G. 'Sniffin' Sticks': olfactory performance assessed by the combined testing of odor identification, odor discrimination and olfactory threshold. Chem Senses. 1997; 22:39-52.

8 Le Berre E, Thomas-Danguin T, Jarmuzek E, Béno N, Etiévant P, Prescott J. Learning influences the perception of odor mixtures. Chem Percept. 2010;3(3-4):156-66.

9 Konstantinidis I, Printza A, Genetzaki S, Mamali K, Kekes G, Constantinidis J. Cultural adaptation of an olfactory identification test: the Greek version of Sniffin' Sticks. Rhinology. 2008;46(4):292-6.

10 Kobal G, Klimek L, Wolfensberger M, Gudziol H, Temmel A, Owen CM, et al. Multicenter investigation of 1036 subjects using a standard method for the assessment of olfactory function combining tests of odor identification, odor discrimination, and olfactory thresholds. Eur Arch Otorhinolaryngol. 2000; 257:205-11

11 Rumeau C, Nguyen DT, Jankowski R. How to assess olfactory performance with the Sniffin' Sticks test $\left({ }^{\circ}\right)$. Eur Ann Otorhinolaryngol Head Neck Dis. 2016;133(3):203-6.
12 Hummel T, Kobal G, Gudziol H, Mackay-Sim A. Normative data for the "Sniffin" Sticks including tests of odor identification, odor discrimination, and olfactory thresholds: An upgrade based on a group of more than 3,000 subjects. Eur Arch Otorhinolaryngol. 2007; 264(3):237-43.

13 Oleszkiewicz A, Schriever VA, Croy I, Hähner A, Hummel T. Updated Sniffin' Sticks normative data based on an extended sample of 9139 subjects. Eur Arch Otorhinolaryngol. 2019;276:719-28.

14 Sai-Guan L, Husain S, Zahedi FD, Ahmad N, Gendeh BS. Cultural adaptation of sniffin' Sticks smell identification test: the Malaysian version. Iran J Otorhinolaryngol. 2020; 32(111):213-22.

15 Ribeiro JC, Simões J, Silva F, Silva D D, Hummel C, Hummel T, et al. Cultural adaptation of the Portuguese version of the "Sniffin' Sticks" smell test: Reliability, validity, and normative data. PLOS One. 2016 Feb 10; 11(2):e0148937.

16 Vickers KL, Breslin K, Roalf DR, Kamath V, Xie SX, Moberg PJ, et al. Older adult normative data for the Sniffin' Sticks odor identification test. Arch Clin Neuropsychol. 2019;34: 254-8.

17 Schriever VA, Mori E, Petters W, Boerner C, Smitka M, Hummel T. The "Sniffin' Kids" test: a 14-item odor identification test for children. PLOS ONE. 2014;309(6):e101086.
18 Sorokowska A, Schriever VA, Gudziol V, Hummel C, Hähner A, Iannilli E, et al. Changes of olfactory abilities in relation to age: odor identification in more than 1400 people aged 4 to 80 years. Eur Arch Otorhinolaryngol. 2015;272:1937-44.

19 Gellrich J, Stetzler C, Oleszkiewicz A, Hummel T, Schriever VA. Olfactory threshold and odor discrimination ability in children - evaluation of a modified "Sniffin' Sticks" test. Sci Rep. 2017;7(1):1928-8.

20 Schriever VA, Agosin E, Altundag A, Avni H, Cao Van HC, Cornejo C, et al. Development of an international odor identification test for children: the universal sniff test. J Pediatr. 2018;198:265-72.e3.

21 Cameron EL, Doty RL. Odor identification testing in children and young adults using the smell wheel. Int J Pediatr Otorhinolaryngol. 2013;77:346-50.

22 Doty RL, Petersen I, Mensah N, Christensen $\mathrm{K}$. Genetic and environmental influences on odor identification ability in the very old. Psychol Aging. 2011;26:864-71.

23 Fornazieri MA, dos Santos CA, Bezerra TFP, Pinna Fde R, Voegels RL, Doty RL. Development of normative data for the Brazilian adaptation of the University of Pennsylvania smell identification test. Chem Senses. 2015; 40:141-9.

24 Schriever VA, Zscheile L, Gellrich J, Hummel T. Odor identification performance in children aged 3-6 years. Pediatr Res. 2021;89(5): 1304-9. 